\title{
Periodic solutions of planar Hamiltonian systems with asymmetric nonlinearities
}

Zaihong Wang ${ }^{1 *}$ and Tiantian $\mathrm{Ma}^{2}$

${ }^{*}$ Correspondence:
zhwang@cnu.edu.cn
${ }^{1}$ School of Mathematical Sciences,
Capital Normal University, Beijing,
100048, People's Republic of China
Full list of author information is
available at the end of the article

${ }^{\text {*Correspondence: }}$

zhwang@cnu.edu.cn

School of Mathematical sciences,

100048 , People's Republic of China

available at the end of the article

\begin{abstract}
In this paper, we look for periodic solutions of planar Hamiltonian systems$$
\left\{\begin{array}{l}
x^{\prime}=f(y)+p_{1}(t, y), \\
y^{\prime}=-g(x)+p_{2}(t, x) .
\end{array}\right.
$$

By using the Poincaré-Birkhoff twist theorem, we prove the existence and multiplicity of periodic solutions of the given system when $f$ satisfies an asymmetric condition and the related time map satisfies an oscillating condition.
\end{abstract}

MSC: $34 C 11 ; 34 C 15 ; 34 C 25$

Keywords: asymmetric nonlinearity; periodic solution; Poincaré-Birkhoff twist theorem

\section{Introduction}

In this paper, we are concerned with the existence and multiplicity of periodic solutions of planar Hamiltonian systems

$$
\left\{\begin{array}{l}
x^{\prime}=f(y)+p_{1}(t, y), \\
y^{\prime}=-g(x)+p_{2}(t, x),
\end{array}\right.
$$

where $f, g: \mathbf{R} \rightarrow \mathbf{R}$ are continuous, $p_{i}: \mathbf{R}^{2} \rightarrow \mathbf{R}(i=1,2)$ are continuous and $2 \pi$-periodic with the first variable $t$.

In the case when $f(y) \equiv y, p_{1}(t, y) \equiv 0$ and $p_{2}(t, x)=p(t)$, system (1.1) becomes

$$
\left\{\begin{array}{l}
x^{\prime}=y, \\
y^{\prime}=-g(x)+p(t),
\end{array}\right.
$$

which is equivalent to the differential equation

$$
x^{\prime \prime}+g(x)=p(t)
$$

The existence and multiplicity of periodic solutions of Eq. (1.2) have been widely studied in the literature (see [1-5] and the references therein). Recently, the periodic solutions of 
planar Hamiltonian systems have been studied with an increasing interest (see [6-11]). In [11], Fonda and Sfecci studied the periodic solutions of the planar Hamiltonian systems of the type

$$
x^{\prime}=g_{1}(t, y), \quad y^{\prime}=-g_{2}(t, x) .
$$

Assume that the following conditions hold:

$$
\begin{aligned}
& a_{+} \leq \liminf _{y \rightarrow+\infty} \frac{g_{1}(t, y)}{y} \leq \limsup _{y \rightarrow+\infty} \frac{g_{1}(t, y)}{y} \leq a^{+}, \\
& a_{-} \leq \liminf _{y \rightarrow-\infty} \frac{g_{1}(t, y)}{y} \leq \limsup _{y \rightarrow-\infty} \frac{g_{1}(t, y)}{y} \leq a^{-},
\end{aligned}
$$

and

$$
\begin{aligned}
& b_{+} \leq \liminf _{x \rightarrow+\infty} \frac{g_{2}(t, x)}{x} \leq \limsup _{x \rightarrow+\infty} \frac{g_{2}(t, x)}{x} \leq b^{+}, \\
& b_{-} \leq \liminf _{x \rightarrow-\infty} \frac{g_{2}(t, x)}{x} \leq \limsup _{x \rightarrow-\infty} \frac{g_{2}(t, x)}{x} \leq b^{-},
\end{aligned}
$$

where $a^{ \pm}, a_{ \pm}, b^{ \pm}$and $b_{ \pm}$are positive constants. It was proved in [11] that system (1.3) has at least one $2 \pi$-periodic solution provided that there exists an integer $n>0$ such that

$$
\left(\frac{1}{\sqrt{a_{+}}}+\frac{1}{\sqrt{a_{-}}}\right)\left(\frac{1}{\sqrt{b_{+}}}+\frac{1}{\sqrt{b_{-}}}\right)<\frac{4}{n}
$$

and

$$
\left(\frac{1}{\sqrt{a^{+}}}+\frac{1}{\sqrt{a^{-}}}\right)\left(\frac{1}{\sqrt{b^{+}}}+\frac{1}{\sqrt{b^{-}}}\right)>\frac{4}{n+1} .
$$

In the present paper, we shall deal with the periodic solutions of system (1.1) when the nonresonant conditions (1.4) and (1.5) do not hold. Assume the following conditions hold:

$\left(h_{1}\right) g$ satisfies $\lim _{|x| \rightarrow+\infty} \operatorname{sgn}(x) g(x)=+\infty$;

$\left(h_{2}\right)$ there exists a constant $L>0$ such that, for all $x, y \in \mathbf{R},|g(x)-g(y)| \leq L|x-y|$;

$\left(h_{3}\right)$ the $\operatorname{limits}_{\lim _{|y| \rightarrow+\infty}} \frac{p_{i}(t, y)}{y}=0(i=1,2)$ hold uniformly with respect to $t \in[0,2 \pi]$;

$\left(h_{4}\right)$ there are two positive constants $a$ and $b$ such that

$$
\lim _{y \rightarrow+\infty} \frac{f(y)}{y}=a, \quad \lim _{y \rightarrow-\infty} \frac{f(y)}{y}=b
$$

It is well known that the time map plays an important role in studying the periodic solutions of Eq. (1.2) (see $[1,2,5]$ and the references therein). In this paper, we also use the time map to study the periodic solutions of system (1.1). Let us set

$$
G(x)=\int_{0}^{x} g(s) d s .
$$


Under condition $\left(h_{1}\right)$, we can define the time map

$$
\tau(c)=\int_{w(c)}^{d(c)} \frac{d s}{\sqrt{2(c-G(s))}}
$$

for $c>0$ large enough, where $w(c)$ and $d(c)$ satisfy $w(c)<0<d(c)$ and $G(w(c))=G(d(c))=c$. Assume that the time map $\tau(c)$ satisfies the condition:

( $\tau$ ) There exist a constant $\sigma>0$, an integer $n>0$, and two sequences $\left\{a_{k}\right\}$ and $\left\{b_{k}\right\}$ such that $\lim _{k \rightarrow \infty} a_{k}=+\infty, \lim _{k \rightarrow \infty} b_{k}=+\infty$; and moreover

$$
\tau\left(a_{k}\right)<\frac{2 \pi}{m n}-\sigma, \quad \tau\left(b_{k}\right)>\frac{2 \pi}{m n}+\sigma,
$$

where $m=\frac{1}{\sqrt{a}}+\frac{1}{\sqrt{b}}$ and $a, b$ are given in condition $\left(h_{4}\right)$.

We prove the following theorem.

Theorem 1.1 Assume that conditions $\left(h_{i}\right)(i=1, \ldots, 4)$ and $(\tau)$ hold. Then system (1.1) has infinitely many $2 \pi$-periodic solutions $\left\{\left(x_{k}(t), y_{k}(t)\right)\right\}_{k=1}^{\infty}$ which satisfy

$$
\lim _{k \rightarrow \infty}\left(\min _{t \in R}\left(\left|x_{k}(t)\right|+\left|y_{k}(t)\right|\right)\right)=+\infty
$$

Moreover, for each integer $k \geq 1$, both $x_{k}(t)$ and $y_{k}(t)$ have exactly $2 n$ simple zeros in $[0,2 \pi)$.

From Theorem 1.1 we can obtain the following corollary.

Corollary 1.2 Assume that a, b are two positive constants, $e, p: \mathbf{R} \rightarrow \mathbf{R}$ are continuous and conditions $\left(h_{i}\right)(i=1,2)$ and $(\tau)$ are satisfied. Then the same conclusions of Theorem 1.1 still hold for the system

$$
\left\{\begin{array}{l}
x^{\prime}=a y^{+}-b y^{-}+e(t), \\
y^{\prime}=-g(x)+p(t)
\end{array}\right.
$$

Remark 1.3 From condition $\left(h_{4}\right)$ we know that $f$ can be written in the form

$$
f(y)=a y^{+}-b y^{-}+h(y)
$$

where $h: \mathbf{R} \rightarrow \mathbf{R}$ is continuous and satisfies

$$
\lim _{|y| \rightarrow+\infty} \frac{h(y)}{y}=0 .
$$

Therefore, it suffices for us to prove the main theorem for the system

$$
\left\{\begin{array}{l}
x^{\prime}=a y^{+}-b y^{-}+p_{1}(t, y) \\
y^{\prime}=-g(x)+p_{2}(t, x)
\end{array}\right.
$$

where $p_{i}(i=1,2)$ satisfy condition $\left(h_{3}\right)$. In the case $a=b \neq 1$, by introducing a rescaling of the time $s=a t, u(s)=x\left(\frac{s}{a}\right), v(s)=y\left(\frac{s}{a}\right)$, we find the equivalent system of $(1.1)^{\prime}$ (having the 
classical form)

$$
\left\{\begin{array}{l}
u^{\prime}=v+q_{1}(s, v) \\
v^{\prime}=-\tilde{g}(u)+q_{2}(s, u) .
\end{array}\right.
$$

Such a rescaling cannot be easily applied in the case $a \neq b$ because we do not know when the solution will change its sign.

We finally stress the fact that the proofs of the above results will be given under the additional assumptions that $f$ and $p_{i}(i=1,2)$ are locally Lipschitz continuous with variables $y$ or $x$. It is shown in Section 4 that this requirement is not restrictive and that our results are valid for any continuous functions $f$ and $p_{i}(i=1,2)$.

\section{Basic lemmas}

At first, we consider the auxiliary autonomous system

$$
x^{\prime}=a y^{+}-b y^{-}, \quad y^{\prime}=-g(x) .
$$

The orbits of system (2.1) are curves determined by the equation

$$
\Gamma_{c}: \quad \frac{1}{2} a y^{+2}+\frac{1}{2} b y^{-2}+G(x)=c
$$

where $c$ is an arbitrary constant. We can easily prove the following lemma.

Lemma 2.1 Assume that condition $\left(h_{1}\right)$ holds. Then there exists a constant $c_{0}>0$ such that, for any $c>c_{0}, \Gamma_{c}$ is a closed curve which is star-shaped around the origin $O$.

From Lemma 2.1 we know that, for $c \geq c_{0}$, each $\Gamma_{c}$ intersects with the $x$-axis at two points $(w(c), 0)$ and $(d(c), 0)$, where $w(c)$ and $d(c)$ are continuous and satisfy

$$
w(c)<0<d(c), \quad G(w(c))=G(d(c))=c .
$$

Let $\left(x_{c}(t), y_{c}(t)\right)$ be the solution of system (2.1) lying on the curve $\Gamma_{c}$ with $c \geq c_{0}$. Obviously, $\left(x_{c}(t), y_{c}(t)\right)$ is periodic. Let us denote by $T(c)$ the least period of $\left(x_{c}(t), y_{c}(t)\right)$. From the first equation of (2.1) and (2.2) we have that

$$
T(c)=\left(\frac{1}{\sqrt{a}}+\frac{1}{\sqrt{b}}\right) \int_{w(c)}^{d(c)} \frac{d s}{\sqrt{2(c-G(s))}} .
$$

By the definition, $T(c)$ is continuous for $c \geq c_{0}$.

Now we perform some phase-plane analysis for system $(1.1)^{\prime}$. Let $(x(t), y(t))=\left(x\left(t, x_{0}, y_{0}\right)\right.$, $\left.y\left(t, x_{0}, y_{0}\right)\right)$ be the solution of system $(1.1)^{\prime}$ satisfying the initial condition

$$
x(0)=x_{0}, \quad y(0)=y_{0} .
$$

Lemma 2.2 Assume that conditions $\left(h_{i}\right)(i=1,2,3)$ hold. Then each solution $(x(t), y(t))$ of system (1.1)' exists uniquely on the whole $t$-axis. 
Proof The proof follows directly from the fact that the nonlinearities are locally Lipschitz continuous and all have at most linear growth.

According to Lemma 2.2, the Poincaré map $P: \mathbf{R}^{2} \rightarrow \mathbf{R}^{2}$ is well defined by

$$
P:\left(x_{0}, y_{0}\right) \rightarrow\left(x_{1}, y_{1}\right)=\left(x\left(2 \pi, x_{0}, y_{0}\right), y\left(2 \pi, x_{0}, y_{0}\right)\right)
$$

Clearly, the Poincaré map $P$ is an area-preserving homeomorphism. The fixed points of $P$ correspond to the $2 \pi$ periodic solutions of system $(1.1)^{\prime}$.

Now, we take the polar coordinates transformation $x=r \cos \theta, y=r \sin \theta$ to system (1.1)'. Under this transformation, system $(1.1)^{\prime}$ becomes

$$
\left\{\begin{aligned}
\frac{d r}{d t}= & r\left(a \sin ^{+} \theta-b \sin ^{-} \theta\right) \cos \theta-g(r \cos \theta) \sin \theta+p_{1}(t, r \sin \theta) \cos \theta \\
& +p_{2}(t, r \cos \theta) \sin \theta \\
\frac{d \theta}{d t}= & -\left(a \sin ^{+} \theta-b \sin ^{-} \theta\right) \sin \theta-\frac{1}{r} g(r \cos \theta) \cos \theta-\frac{1}{r} p_{1}(t, r \sin \theta) \sin \theta \\
& +\frac{1}{r} p_{2}(t, r \cos \theta) \cos \theta
\end{aligned}\right.
$$

Denote by $(r(t), \theta(t))=\left(r\left(t, r_{0}, \theta_{0}\right), \theta\left(t, r_{0}, \theta_{0}\right)\right)$ the solution of (2.3) with the initial value

$$
r(0)=r_{0}, \quad \theta(0)=\theta_{0},
$$

with $x_{0}=r_{0} \cos \theta_{0}, y_{0}=r_{0} \sin \theta_{0}$. Clearly, the Poincaré map $P$ can be written in the polar coordinate form $P:\left(r_{0}, \theta_{0}\right) \rightarrow\left(r^{*}, \theta^{*}\right)$ with

$$
r^{*}=r\left(2 \pi, r_{0}, \theta_{0}\right), \quad \theta^{*}=\theta\left(2 \pi, r_{0}, \theta_{0}\right)+2 l \pi,
$$

where $l$ is an arbitrary integer.

Applying the polar coordinate transformation $x=\rho \cos \varphi, y=\rho \sin \varphi$ to system (2.1), we get

$$
\left\{\begin{array}{l}
\frac{d \rho}{d t}=\rho\left(a \sin ^{+} \varphi-b \sin ^{-} \varphi\right) \cos \varphi-g(\rho \cos \varphi) \sin \varphi \\
\frac{d \varphi}{d t}=-\left(a \sin ^{+} \varphi-b \sin ^{-} \varphi\right) \sin \varphi-\frac{1}{\rho} g(\rho \cos \varphi) \cos \varphi .
\end{array}\right.
$$

Denote by $(\rho(t), \varphi(t))=\left(\rho\left(t, \rho_{0}, \varphi_{0}\right), \varphi\left(t, \rho_{0}, \varphi_{0}\right)\right)$ the solution of (2.4) satisfying the initial value

$$
\rho(0)=\rho_{0}, \quad \varphi(0)=\varphi_{0} .
$$

Using conditions $\left(h_{i}\right)(i=1,2,3)$, it is not hard to prove the following lemma.

Lemma 2.3 Assume that conditions $\left(h_{i}\right)(i=1,2,3)$ hold. Then there exist constants $\gamma>1$ and $R_{0}>0$ such that

$$
\frac{r_{0}}{\gamma} \leq r(t) \leq \gamma r_{0}, \quad \forall t \in[0,2 \pi], r_{0} \geq R_{0}
$$

In particular, under conditions $\left(h_{i}\right)(i=1,2), \rho(t)$ satisfies the inequality

$$
\frac{\rho_{0}}{\gamma} \leq \rho(t) \leq \gamma \rho_{0}, \quad \forall t \in[0,2 \pi], \rho_{0} \geq R_{0}
$$


Lemma 2.4 Assume that conditions $\left(h_{i}\right)(i=1,2,3)$ hold, and let

$$
\Phi\left(r_{0}, \theta_{0}\right)=\varphi\left(2 \pi, r_{0}, \theta_{0}\right)-\theta_{0} ; \quad \Theta\left(r_{0}, \theta_{0}\right)=\theta\left(2 \pi, r_{0}, \theta_{0}\right)-\theta_{0}
$$

Then, for any sufficiently small $\varepsilon$, there exists a positive constant $\zeta$ such that

$$
\left|\Phi\left(r_{0}, \theta_{0}\right)-\Theta\left(r_{0}, \theta_{0}\right)\right| \leq \varepsilon \quad \text { for } r_{0} \geq \zeta
$$

Proof Let $(\bar{x}(t), \bar{y}(t))=\left(\bar{x}\left(t, x_{0}, y_{0}\right), \bar{y}\left(t, x_{0}, y_{0}\right)\right)$ be the solution of $(2.1)$ with $(\bar{x}(0), \bar{y}(0))=$ $\left(x_{0}, y_{0}\right)$. It is noted that $(x(t), y(t))=\left(x\left(t, x_{0}, y_{0}\right), y\left(t, x_{0}, y_{0}\right)\right)$ is a solution of system $(1.1)^{\prime}$ with $(x(0), y(0))=\left(x_{0}, y_{0}\right)$. Set

$$
\begin{aligned}
& u(t)=u\left(t, x_{0}, y_{0}\right)=x\left(t, x_{0}, y_{0}\right)-\bar{x}\left(t, x_{0}, y_{0}\right), \\
& v(t)=v\left(t, x_{0}, y_{0}\right)=y\left(t, x_{0}, y_{0}\right)-\bar{y}\left(t, x_{0}, y_{0}\right) .
\end{aligned}
$$

Then we have

$$
\begin{aligned}
& \frac{d u(t)}{d t}=a\left[y^{+}(t)-\bar{y}^{+}(t)\right]-b\left[y^{-}(t)-\bar{y}^{-}(t)\right]+p_{1}(t, y(t)), \\
& \frac{d v(t)}{d t}=-[g(x(t))-g(\bar{x}(t))]+p_{2}(t, x(t)) .
\end{aligned}
$$

Let $d(t)=\sqrt{u^{2}(t)+v^{2}(t)}$. Then we get

$$
d^{\prime}(t) \leq \delta d(t)+\left|p_{1}(t, y(t))\right|+\left|p_{2}(t, x(t))\right|
$$

with $\delta=\frac{1}{2}(\mu+L), \mu=\max \{a, b\}$. From condition $\left(h_{3}\right)$ we have that, for any sufficiently small $\eta>0$, there exists $c_{\eta}>0$ such that

$$
\left|p_{1}(t, y)\right| \leq \eta|y|+c_{\eta}, \quad \forall(t, y) \in \mathbf{R}^{2}
$$

and

$$
\left|p_{2}(t, x)\right| \leq \eta|x|+c_{\eta}, \quad \forall(t, x) \in \mathbf{R}^{2}
$$

Therefore, we obtain

$$
d^{\prime}(t) \leq \delta d(t)+\eta(|x(t)|+|y(t)|)+2 c_{\eta} .
$$

Solving this inequality, we get

$$
d(t) \leq \eta e^{2 \pi \delta} \int_{0}^{2 \pi}(|x(t)|+|y(t)|) d t+A_{\eta} \leq \sqrt{2} \eta e^{2 \pi \delta} \int_{0}^{2 \pi} \sqrt{x^{2}(t)+y^{2}(t)} d t+A_{\eta}
$$

where $A_{\eta}=\frac{2 c_{\eta} e^{2 \pi \delta}}{\delta}$. It follows from Lemma 2.3 that, for $t \in[0,2 \pi]$,

$$
d(t) \leq \eta \beta r_{0}+A_{\eta},
$$


where $\beta=2 \sqrt{2} \pi \gamma e^{2 \pi \delta}$. Write $\psi(t)=\psi\left(t, r_{0}, \theta_{0}\right)=\varphi\left(t, r_{0}, \theta_{0}\right)-\theta\left(t, r_{0}, \theta_{0}\right)$. Clearly, if $|\psi(t)|<$ $\pi$, then $\psi(t)$ is just the angle between the vectors $(x(t), y(t))$ and $(\bar{x}(t), \bar{y}(t))$. Hence, we have

$$
\cos \psi(t)=\frac{r^{2}(t)+\rho^{2}(t)-d^{2}(t)}{2 r(t) \rho(t)} \geq 1-\frac{d^{2}(t)}{2 r(t) \rho(t)} .
$$

It follows that

$$
\sin ^{2} \frac{\psi(t)}{2} \leq \frac{d^{2}(t)}{4 r(t) \rho(t)}
$$

According to Lemma 2.3, we have that if $\eta$ is sufficiently small and $r_{0}$ is large enough, then

$$
\left|\sin \frac{\psi(t)}{2}\right| \leq \frac{d(t)}{2 \sqrt{r(t) \rho(t)}} \leq \frac{\gamma\left(\eta \beta r_{0}+A_{\eta}\right)}{2 r_{0}} \leq \frac{\varepsilon}{4} .
$$

Since $\psi(0)=0$ and $\psi(t)$ varies continuously as $t$ increases from 0 to $2 \pi$, we have

$$
|\psi(t)| \leq 4\left|\sin \frac{\psi(t)}{2}\right| \leq \varepsilon
$$

Consequently, we have that there exists $\zeta>0$ such that, for $r_{0} \geq \zeta$,

$$
\left|\Phi\left(r_{0}, \theta_{0}\right)-\Theta\left(r_{0}, \theta_{0}\right)\right| \leq \varepsilon, \quad \forall r_{0} \geq \zeta
$$

Lemma 2.5 Assume that conditions $\left(h_{i}\right)(i=1,2)$ and $(\tau)$ hold. Then there exists a constant $\omega>0$ such that, for $t \in \mathbf{R}$ and $k$ large enough,

$$
\varphi^{\prime}(t) \leq-\omega
$$

with $\left(\rho_{0} \cos \varphi_{0}, \rho_{0} \sin \varphi_{0}\right) \in \Gamma_{a_{k}}$ or $\left(\rho_{0} \cos \varphi_{0}, \rho_{0} \sin \varphi_{0}\right) \in \Gamma_{b_{k}}$.

Proof From the definition of $T(c)$ and condition $(\tau)$ we know that, for each $k \in \mathbf{N}$,

$$
T\left(a_{k}\right) \leq \frac{2 \pi}{n}-m \sigma ; \quad T\left(b_{k}\right) \geq \frac{2 \pi}{n}+m \sigma .
$$

In what follows, without loss of generality, we assume that the sequence $\left\{T\left(b_{k}\right)\right\}$ is bounded. Otherwise, we can replace the sequence $\left\{T\left(b_{k}\right)\right\}$ with a bounded one because $T(c)$ is continuous for $c$ large enough. We shall only deal with the first case, and the second one can be proved similarly. Let us set

$$
d_{k}=d\left(a_{k}\right), \quad w_{k}=w\left(a_{k}\right)
$$

Obviously, $d_{k} \rightarrow+\infty, w_{k} \rightarrow-\infty$ as $k \rightarrow \infty$. Next, we prove that there exist two positive constants $v_{i}(i=1,2)$ such that

$$
\liminf _{k \rightarrow \infty} \frac{g\left(d_{k}\right)}{d_{k}}=v_{1} ; \quad \liminf _{k \rightarrow \infty} \frac{g\left(w_{k}\right)}{w_{k}}=v_{2} .
$$


Assume by contradiction that

$$
\liminf _{k \rightarrow \infty} \frac{g\left(d_{k}\right)}{d_{k}}=0 .
$$

Then there exists a subsequence of $\left\{d_{k}\right\}$ (we still denote it by $\left\{d_{k}\right\}$ ) such that

$$
\lim _{k \rightarrow \infty} \frac{g\left(d_{k}\right)}{d_{k}}=0
$$

Set

$$
\frac{g\left(d_{k}\right)}{d_{k}}=\varepsilon_{k}
$$

We have that $\varepsilon_{k} \rightarrow 0$ as $k \rightarrow \infty$. From condition $\left(h_{2}\right)$ we know that, for $0<x \leq d_{k}$,

$$
\begin{aligned}
\left|\frac{g(x)}{x}-\varepsilon_{k}\right| & =\left|\frac{g(x)}{x}-\frac{g\left(d_{k}\right)}{d_{k}}\right| \leq \frac{\left|g\left(d_{k}\right)-g(x)\right|}{x}+\frac{\left|g\left(d_{k}\right)\left(d_{k}-x\right)\right|}{d_{k} x} \\
& \leq \frac{L\left(d_{k}-x\right)}{x}+\left(L+\frac{|g(0)|}{d_{k}}\right) \frac{\left(d_{k}-x\right)}{x} .
\end{aligned}
$$

For simplicity, we assume $g(0)=0$. Then we get that, for $0<x \leq d_{k}$,

$$
\left|\frac{g(x)}{x}-\varepsilon_{k}\right| \leq \frac{2 L\left(d_{k}-x\right)}{x} .
$$

Consequently, we have that, for $0 \leq x \leq d_{k}$,

$$
g(x) \leq \varepsilon_{k} x+2 L\left(d_{k}-x\right) .
$$

It follows that, for $0 \leq x \leq d_{k}$,

$$
\begin{aligned}
G\left(d_{k}\right)-G(x) & =\int_{x}^{d_{k}} g(s) d s \leq \int_{x}^{d_{k}}\left[\varepsilon_{k} x+2 L\left(d_{k}-x\right)\right] d x \\
& =\frac{1}{2} \varepsilon_{k}\left(d_{k}^{2}-x^{2}\right)+L\left(d_{k}-x\right)^{2} .
\end{aligned}
$$

From the definition of $T$ we have that

$$
\begin{aligned}
& T\left(a_{k}\right)=m \int_{w_{k}}^{d_{k}} \frac{d x}{\sqrt{2\left(G\left(d_{k}\right)-G(x)\right)}} \geq m \int_{0}^{d_{k}} \frac{d x}{\sqrt{2\left(G\left(d_{k}\right)-G(x)\right)}} \\
& \geq m \int_{0}^{d_{k}} \frac{d x}{\sqrt{\varepsilon_{k}\left(d_{k}^{2}-x^{2}\right)+2 L\left(d_{k}-x\right)^{2}}} \\
& =m \int_{0}^{1} \frac{d t}{\sqrt{\varepsilon_{k}\left(1-t^{2}\right)+2 L(1-t)^{2}}} \text {. }
\end{aligned}
$$

Since

$$
\liminf _{k \rightarrow \infty} \int_{0}^{1} \frac{d t}{\sqrt{\varepsilon_{k}\left(1-t^{2}\right)+2 L(1-t)^{2}}} \geq \frac{1}{\sqrt{2 L}} \int_{0}^{1} \frac{d t}{1-t}=+\infty,
$$


we have that

$$
\lim _{k \rightarrow \infty} T\left(a_{k}\right)=+\infty
$$

This is a contradiction because $T\left(a_{k}\right)$ is a bounded sequence. Therefore, there exists a constant $v_{1}>0$ such that

$$
\liminf _{k \rightarrow \infty} \frac{g\left(d_{k}\right)}{d_{k}}=v_{1}
$$

Similarly, there exists $v_{2}>0$ such that

$$
\liminf _{k \rightarrow \infty} \frac{g\left(w_{k}\right)}{w_{k}}=v_{2} .
$$

From condition $\left(h_{1}\right)$ and (2.5), (2.6) we know that there exists sufficiently small $\varepsilon_{0}>0$ such that, for $k$ large enough and $x \in\left[\left(1-\varepsilon_{0}\right) d_{k}, d_{k}\right]$,

$$
\frac{g(x)}{x} \geq \frac{g\left(d_{k}\right)}{d_{k}}-\frac{2 L\left(d_{k}-x\right)}{x} \geq \frac{1}{2} v_{1}-\frac{2 \varepsilon_{0} L}{1-\varepsilon_{0}} \geq \frac{1}{4} v_{1} .
$$

Therefore, if $\rho(t) \cos \varphi(t) \in\left[\left(1-\varepsilon_{0}\right) d_{k}, d_{k}\right]$, then we have

$$
\varphi^{\prime}(t) \leq-\left(a \sin ^{+} \varphi(t)-b \sin ^{-} \varphi(t)\right) \sin \varphi(t)-\frac{1}{4} v_{1} \cos ^{2} \varphi(t) \leq-\omega_{1}
$$

where $\omega_{1}=\min \left\{a, b, \frac{1}{4} v_{1}\right\}$. Next, we deal with the case $\rho(t) \cos \varphi(t) \in\left[0,\left(1-\varepsilon_{0}\right) d_{k}\right]$. Set $x_{k}=\left(1-\varepsilon_{0}\right) d_{k}$. Assume that the line $x=x_{k}$ intersects with the curve $\Gamma_{a_{k}}$ at two points $\left(x_{k}, y_{k}^{+}\right)$and $\left(x_{k}, y_{k}^{-}\right)$with $y_{k}^{-}<0<y_{k}^{+}$. Then we have

$$
\frac{1}{2} a y_{k}^{+2}+G\left(x_{k}\right)=a_{k}=G\left(d_{k}\right), \quad \frac{1}{2} b y_{k}^{-2}+G\left(x_{k}\right)=a_{k}=G\left(d_{k}\right) .
$$

Therefore, we get

$$
y_{k}^{+}=\sqrt{\frac{2}{a}\left(G\left(d_{k}\right)-G\left(\left(1-\varepsilon_{0}\right) d_{k}\right)\right)}, \quad y_{k}^{-}=-\sqrt{\frac{2}{b}\left(G\left(d_{k}\right)-G\left(\left(1-\varepsilon_{0}\right) d_{k}\right)\right)} .
$$

From (2.7) we have

$$
\frac{y_{k}^{+}}{x_{k}}=\frac{\sqrt{\frac{2}{a}\left(G\left(d_{k}\right)-G\left(\left(1-\varepsilon_{0}\right) d_{k}\right)\right)}}{\left(1-\varepsilon_{0}\right) d_{k}} \geq \sqrt{\frac{\varepsilon_{0} \nu_{1}}{2 a\left(1-\varepsilon_{0}\right)}}
$$

and

$$
\frac{y_{k}^{-}}{x_{k}}=-\frac{\sqrt{\frac{2}{b}\left(G\left(d_{k}\right)-G\left(\left(1-\varepsilon_{0}\right) d_{k}\right)\right)}}{\left(1-\varepsilon_{0}\right) d_{k}} \leq-\sqrt{\frac{\varepsilon_{0} v_{1}}{2 b\left(1-\varepsilon_{0}\right)}} .
$$

Set

$$
\beta^{+}=\arctan \sqrt{\frac{\varepsilon_{0} v_{1}}{2 a\left(1-\varepsilon_{0}\right)}}, \quad \beta^{-}=\arctan \sqrt{\frac{\varepsilon_{0} v_{1}}{2 b\left(1-\varepsilon_{0}\right)}} .
$$


From condition $\left(h_{1}\right)$ we know that there exists $A>0$ such that $g(x) \geq 0$ for $x \geq A$. If $\rho(t) \cos \varphi(t) \in\left[A,\left(1-\varepsilon_{0}\right) d_{k}\right]$ and $\rho(t) \sin \varphi(t) \geq 0$, then we have

$$
\varphi^{\prime}(t) \leq-a \sin ^{2} \beta^{+}-\frac{1}{\rho} g(\rho \cos \varphi) \cos \varphi \leq-a \sin ^{2} \beta^{+} .
$$

If $\rho(t) \cos \varphi(t) \in[0, A]$ and $\rho(t) \sin \varphi(t) \geq 0$, then we have that, for $\rho_{0}$ large enough,

$$
\varphi^{\prime}(t) \leq-a \sin ^{2} \beta^{+}-\frac{1}{\rho} g(\rho \cos \varphi) \cos \varphi \leq-\frac{1}{2} a \sin ^{2} \beta^{+} .
$$

Similarly, we have that, if $\rho(t) \cos \varphi(t) \in\left[A,\left(1-\varepsilon_{0}\right) d_{k}\right]$ and $\rho(t) \sin \varphi(t) \leq 0$, then we have

$$
\varphi^{\prime}(t) \leq-b \sin ^{2} \beta^{-}-\frac{1}{\rho} g(\rho \cos \varphi) \cos \varphi \leq-b \sin ^{2} \beta^{-} .
$$

If $\rho(t) \cos \varphi(t) \in[0, A]$ and $\rho(t) \sin \varphi(t) \leq 0$, then we have that, for $\rho_{0}$ large enough,

$$
\varphi^{\prime}(t) \leq-b \sin ^{2} \beta^{-}-\frac{1}{\rho} g(\rho \cos \varphi) \cos \varphi \leq-\frac{1}{2} b \sin ^{2} \beta^{-} .
$$

In conclusion, we have proved that there exists $\omega_{2}>0$ such that

$$
\varphi^{\prime}(t) \leq-\omega_{2}
$$

with $\left(\rho_{0} \cos \varphi_{0}, \rho_{0} \sin \varphi_{0}\right) \in \Gamma_{a_{k}}, \rho(t) \cos \varphi(t) \geq 0$ and $k$ large enough. Similarly, we can prove that there exists $\omega_{2}^{\prime}>0$ such that

$$
\varphi^{\prime}(t) \leq-\omega_{2}^{\prime}
$$

with $\left(\rho_{0} \cos \varphi_{0}, \rho_{0} \sin \varphi_{0}\right) \in \Gamma_{a_{k}}, \rho(t) \cos \varphi(t) \leq 0$ and $k$ large enough. Let us set $\omega=$ $\min \left\{\omega_{2}, \omega_{2}^{\prime}\right\}$. Then we have that

$$
\varphi^{\prime}(t) \leq-\omega
$$

with $\left(\rho_{0} \cos \varphi_{0}, \rho_{0} \sin \varphi_{0}\right) \in \Gamma_{a_{k}}$ and $k$ large enough.

Lemma 2.6 Assume that conditions $\left(h_{i}\right)(i=1,2)$ and $(\tau)$ hold, and let $\Phi\left(\rho_{0}, \varphi_{0}\right)=$ $\varphi\left(2 \pi, \rho_{0}, \varphi_{0}\right)-\varphi_{0}$. Then there exist two positive constants $\delta$ and $\varrho_{0}$ such that

$$
\begin{array}{lll}
\Phi\left(\rho_{0}, \varphi_{0}\right)<-2 n \pi-\delta, & \left(\rho_{0} \cos \varphi_{0}, \rho_{0} \sin \varphi_{0}\right) \in \Gamma_{a_{k}}, & a_{k} \geq \varrho_{0} \\
\Phi\left(\rho_{0}, \varphi_{0}\right)>-2 n \pi+\delta, & \left(\rho_{0} \cos \varphi_{0}, \rho_{0} \sin \varphi_{0}\right) \in \Gamma_{b_{k}}, & b_{k} \geq \varrho_{0} .
\end{array}
$$

Proof From Lemma 2.5 we have that there exists $\varrho_{0}>0$ such that, for $a_{k} \geq \varrho_{0}$ or $b_{k} \geq \varrho_{0}$,

$$
\varphi^{\prime}(t) \leq-\omega, \quad\left(\rho_{0} \cos \varphi_{0}, \rho_{0} \sin \varphi_{0}\right) \in \Gamma_{a_{k}} \quad \text { or } \quad\left(\rho_{0} \cos \varphi_{0}, \rho_{0} \sin \varphi_{0}\right) \in \Gamma_{b_{k}} .
$$

Write $\Phi\left(\rho_{0}, \varphi_{0}\right)=-2 l \pi-\phi$, where $l \geq 0$ is an integer, $0 \leq \phi<2 \pi$. Let us denote by $t_{\phi}$ the time for $\varphi(t)$ to decrease from $-2 l \pi$ to $-2 l \pi-\phi$. If $\left(\rho_{0} \cos \varphi_{0}, \rho_{0} \sin \varphi_{0}\right) \in \Gamma_{a_{k}}$, then we have

$$
2 \pi=l T\left(a_{k}\right)+t_{\phi}=\operatorname{lm} \tau\left(a_{k}\right)+t_{\phi}
$$


Since $t_{\phi} \leq T\left(a_{k}\right)=m \tau\left(a_{k}\right)$, we have

$$
2 \pi=\operatorname{lm} \tau\left(a_{k}\right)+t_{\phi} \leq(l+1) m \tau\left(a_{k}\right) \leq(l+1)\left(\frac{2 \pi}{n}-m \sigma\right) .
$$

It follows that $l \geq n$. If $l \geq n+1$, then we have

$$
\Phi\left(\rho_{0}, \varphi_{0}\right)=-2 l \pi-\phi \leq-2(n+1) \pi .
$$

If $l=n$, then we have

$$
t_{\phi}=2 \pi-n m \tau\left(a_{k}\right) \geq 2 \pi-n\left(\frac{2 \pi}{n}-m \sigma\right) \geq n m \sigma .
$$

Therefore, we get

$$
-\phi=\int_{n T\left(a_{k}\right)}^{n T\left(a_{k}\right)+t_{\phi}} \varphi^{\prime}(t) d t \leq-n m \sigma \omega .
$$

Furthermore,

$$
\Phi\left(\rho_{0}, \varphi_{0}\right) \leq-2 n \pi-n m \sigma \omega .
$$

Set $\delta=\min \{2 \pi, n m \sigma \omega\}$. Then we have

$$
\Phi\left(\rho_{0}, \varphi_{0}\right) \leq-2 n \pi-\delta, \quad\left(\rho_{0} \cos \varphi_{0}, \rho_{0} \sin \varphi_{0}\right) \in \Gamma_{a_{k}}, \quad a_{k} \geq \varrho_{0} .
$$

Similarly, we can prove

$$
\Phi\left(\rho_{0}, \varphi_{0}\right) \geq-2 n \pi+\delta, \quad\left(\rho_{0} \cos \varphi_{0}, \rho_{0} \sin \varphi_{0}\right) \in \Gamma_{b_{k}}, \quad b_{k} \geq \varrho_{0} .
$$

\section{Proof of the main theorem}

At first, we recall a generalized version of the Poincaré-Birkhoff fixed point theorem by Rebelo [12].

A generalized form of the Poincaré-Birkhoff fixed point theorem. Let $\mathcal{A} \subset \mathbf{R}^{2}$ be an annular region bounded by two strictly star-shaped curves around the origin, $\Gamma_{1}$ and $\Gamma_{2}$, $\Gamma_{1} \subset \operatorname{int}\left(\Gamma_{2}\right)$, where $\operatorname{int}\left(\Gamma_{2}\right)$ denotes the interior domain bounded by $\Gamma_{2}$. Suppose that $F$ : $\overline{\operatorname{int}\left(\Gamma_{2}\right)} \rightarrow R^{2}$ is an area-preserving homeomorphism and $F \mid \mathcal{A}$ admits a lifting, with the standard covering projection $\Pi:(r, \theta) \rightarrow z=(r \cos \theta, r \sin \theta)$, of the form

$$
\tilde{F} \mid \mathcal{A}:(r, \theta) \rightarrow(w(r, \theta), \theta+h(r, \theta)),
$$

where $w$ and $h$ are continuous functions of period $2 \pi$ in the first variable. Correspondingly, for $\tilde{\Gamma}_{1}=\Pi^{-1}\left(\Gamma_{1}\right)$ and $\tilde{\Gamma}_{2}=\Pi^{-1}\left(\Gamma_{2}\right)$, assume the twist condition

$$
h(r, \theta)>0 \quad \text { on } \tilde{\Gamma}_{1} ; \quad h(r, \theta)<0 \quad \text { on } \tilde{\Gamma}_{2} .
$$

Then, $F$ has two fixed points $z_{1}, z_{2}$ in the interior of $\mathcal{A}$ such that

$$
h\left(\Pi^{-1}\left(z_{1}\right)\right)=h\left(\Pi^{-1}\left(z_{2}\right)\right)=0 .
$$


Remark 3.1 The assumption on the star-shaped boundaries of the annulus is a delicate hypothesis. Martins and Ureña [13] showed that the star-shapedness assumption on the interior boundary is not eliminable. Le Calvez and Wang [14] then proved that starshapedness of the exterior boundary should also be imposed, while this assumption was not made in Ding's theorem [15].

Proof of Theorem 1.1 From Lemmas 2.4 and 2.6 we know that there exists an integer $k_{0}>0$ such that, for any $k \geq k_{0}$,

$$
\begin{array}{ll}
\theta\left(2 \pi, r_{0}, \theta_{0}\right)-\theta_{0}<-2 n \pi, & \left(r_{0} \cos \theta_{0}, r_{0} \sin \theta_{0}\right) \in \Gamma_{a_{k}}, \\
\theta\left(2 \pi, r_{0}, \theta_{0}\right)-\theta_{0}>-2 n \pi, & \left(r_{0} \cos \theta_{0}, r_{0} \sin \theta_{0}\right) \in \Gamma_{b_{k}} .
\end{array}
$$

Without loss of generality, we assume that $a_{k}<b_{k}<a_{k+1}$ for $k \geq k_{0}$. Let $D_{k}$ be an annular region with boundary $\Gamma_{a_{k}}$ and $\Gamma_{b_{k}}$. Consider the Poincaré map $P: D_{k} \rightarrow \mathbf{R}^{2}$. Write the Poincaré map in the form

$$
r^{*}=r\left(2 \pi, r_{0}, \theta_{0}\right), \quad \theta^{*}=\theta_{0}+\Theta^{*}\left(r_{0}, \theta_{0}\right)
$$

where $\Theta^{*}\left(r_{0}, \theta_{0}\right)=\theta\left(2 \pi, r_{0}, \theta_{0}\right)-\theta_{0}+2 n \pi$. From (3.1) and (3.2) we know that, for $k \geq k_{0}$,

$$
\begin{array}{ll}
\Theta^{*}\left(r_{0}, \theta_{0}\right)<0, & \left(r_{0} \cos \theta_{0}, r_{0} \sin \theta_{0}\right) \in \Gamma_{a_{k}}, \\
\Theta^{*}\left(r_{0}, \theta_{0}\right)>0, & \left(r_{0} \cos \theta_{0}, r_{0} \sin \theta_{0}\right) \in \Gamma_{b_{k}} .
\end{array}
$$

Therefore, the area-preserving homeomorphism $P$ is twisting on the annulus $D_{k}$. On the other hand, by Lemma 2.1, we have that both $\Gamma_{a_{k}}$ and $\Gamma_{b_{k}}$ are star-shaped with respect to the origin $O$ for $k$ large enough. Consequently, all assumptions of the generalized form of the Poincaré-Birkhoff fixed point theorem are satisfied. Therefore, the Poincaré map $P$ has at least two fixed points in annulus $D_{k}$. Furthermore, system $(1.1)^{\prime}$ has infinitely many $2 \pi$-periodic solutions $\left\{\left(x_{k}(t), y_{k}(t)\right)\right\}_{k=1}^{\infty}$ which satisfy

$$
\lim _{k \rightarrow \infty}\left(\min _{t \in R}\left(\left|x_{k}(t)\right|+\left|y_{k}(t)\right|\right)\right)=+\infty
$$

Clearly, each $2 \pi$-periodic solution $\left(x_{k}(t), y_{k}(t)\right)$ rotates clockwise strictly $n$ turns around the origin in the interval $[0,2 \pi]$. It follows that both $x_{k}(t)$ and $y_{k}(t)$ have exactly $2 n$ simple zeros in $[0,2 \pi)$. Hence, the conclusion of Theorem 1.1 holds.

From Theorem 1.1 we know that the existence of periodic solutions of system (1.1) has tight relation with the asymptotic property of time map $\tau(c)$. In case $g$ is odd, we can easily check condition $(\tau)$. Set

$$
G_{+}=\liminf _{x \rightarrow+\infty} \frac{2 G(x)}{x^{2}}, \quad G^{+}=\limsup _{x \rightarrow+\infty} \frac{2 G(x)}{x^{2}}
$$

From Theorem 3.1 in [1], we have the following lemma. 
Lemma 3.2 Assume that condition $\left(h_{1}\right)$ holds, $g$ is odd and $G_{+}<G^{+}$. Then

$$
\left[\frac{\pi}{\sqrt{G^{+}}}, \frac{\pi}{\sqrt{G_{+}}}\right] \subset\left[\tau_{+}, \tau^{+}\right]
$$

where $\tau_{+}=\liminf _{c \rightarrow+\infty} \tau(c), \tau^{+}=\limsup _{c \rightarrow+\infty} \tau(c)$.

Applying Theorem 1.1 and Lemma 3.2, we can obtain the following corollary.

Corollary 3.3 Assume that conditions $\left(h_{i}\right)(i=1, \ldots, 4)$ hold. Let $g(x)$ be an odd function and $G_{+}<G^{+}$. If

$$
\exists n \in \mathbf{N}, \quad \frac{m^{2} n^{2}}{4} \in\left(G_{+}, G^{+}\right) \quad\left(m=\frac{1}{\sqrt{a}}+\frac{1}{\sqrt{b}}\right)
$$

then system (1.1) has infinitely many $2 \pi$-periodic solutions $\left\{\left(x_{k}(t), y_{k}(t)\right)\right\}_{k=1}^{\infty}$ which satisfy

$$
\lim _{k \rightarrow \infty}\left(\min _{t \in R}\left(\left|x_{k}(t)\right|+\left|y_{k}(t)\right|\right)\right)=+\infty
$$

Moreover, for each integer $k \geq 1$, both $x_{k}(t)$ and $y_{k}(t)$ have exactly $2 n$ simple zeros in $[0,2 \pi)$.

\section{Concluding remarks}

The restrictions on the local Lipschitz conditions of $f$ and $p_{i}(i=1,2)$ made in the proofs of the above sections can be removed. Indeed, Lemmas 2.4 and 2.6 guarantee the applicability of the following non-uniqueness version of the Poincaré-Birkhoff theorem which was proved by Fonda and Ureña in [16]. We now state this theorem for a general Hamiltonian system in $\mathbf{R}^{2 N}$. Let us consider the (time-dependent) Hamiltonian system

$$
\left\{\begin{array}{l}
x^{\prime}=\nabla_{y} H(t, x, y), \\
y^{\prime}=-\nabla_{x} H(t, x, y),
\end{array}\right.
$$

where the continuous function $H: \mathbf{R} \times \mathbf{R}^{N} \times \mathbf{R}^{N} \rightarrow \mathbf{R}, H=H(t, x, y)$ is $T$-periodic in its first variable $t$ and continuously differentiable with respect to $(x, y), x=\left(x_{1}, \ldots, x_{N}\right), y=$ $\left(y_{1}, \ldots, y_{N}\right)$.

We next introduce the definition of rotation number of a continuous path in $\mathbf{R}^{2}$. Let $w$ : $\left[t_{1}, t_{2}\right] \rightarrow \mathbf{R}^{2}$ be a continuous path such that $w(t) \neq(0,0)$ for every $t \in\left[t_{1}, t_{2}\right]$. The rotation number of $w$ around the origin is defined as

$$
\operatorname{Rot}\left(w(t) ;\left[t_{1}, t_{2}\right]\right)=\frac{\theta\left(t_{2}\right)-\theta\left(t_{1}\right)}{2 \pi}
$$

where $\theta:\left[t_{1}, t_{2}\right] \rightarrow \mathbf{R}$ is a continuous determination of the argument along $w$, i.e., $w(t)=$ $|w(t)|(\cos \theta(t), \sin \theta(t))$.

Assume that for each $i=1, \ldots, N$, there are two strictly star-shaped Jordan closed curves around the origin $\Gamma_{1}^{i}, \Gamma_{2}^{i} \subset \mathbf{R}^{2}$ such that

$$
o \in \mathcal{D}\left(\Gamma_{1}^{i}\right) \subset \overline{\mathcal{D}\left(\Gamma_{1}^{i}\right)} \subset \mathcal{D}\left(\Gamma_{2}^{i}\right) .
$$


Here we denote by $\mathcal{D}(\Gamma)$ the open bounded region bounded by the Jordan closed curve $\Gamma$. Consider the generalized annular region

$$
\mathcal{A}=\left[\overline{\mathcal{D}\left(\Gamma_{2}^{1}\right)} \backslash \mathcal{D}\left(\Gamma_{1}^{1}\right)\right] \times \cdots \times\left[\overline{\mathcal{D}\left(\Gamma_{2}^{N}\right)} \backslash \mathcal{D}\left(\Gamma_{1}^{N}\right)\right] \subset \mathbf{R}^{2 N} .
$$

Theorem 4.1 ([16]) Under the framework above, denoting $z_{i}(t)=\left(x_{i}(t), y_{i}(t)\right)$, assume that every solution $z(t)=\left(z_{1}(t), \ldots, z_{N}(t)\right)$ of (4.1) departing from $z(0) \in \partial \mathcal{A}$ is defined on $[0, T]$ and satisfies

$$
z_{i}(t) \neq(0,0) \quad \text { for every } t \in[0, T] \text { and } i=1, \ldots, N \text {. }
$$

Assume that there are integer numbers $v_{1}, \ldots, v_{N} \in \mathbf{Z}$ such that, for each $i=1, \ldots, N$, either

$$
\operatorname{Rot}\left(z_{i}(t) ;[0, T]\right) \begin{cases}<v_{i}, & \text { if } z_{i}(0) \in \Gamma_{1}^{i}, \\ >v_{i}, & \text { if } z_{i}(0) \in \Gamma_{2}^{i},\end{cases}
$$

or

$$
\operatorname{Rot}\left(z_{i}(t) ;[0, T]\right) \begin{cases}>v_{i}, & \text { if } z_{i}(0) \in \Gamma_{1}^{i}, \\ <v_{i}, & \text { if } z_{i}(0) \in \Gamma_{2}^{i} .\end{cases}
$$

Then Hamiltonian system (4.1) has at least $N+1$ distinct $T$-periodic solutions $z^{0}(t), \ldots$, $z^{N}(t)$, with $z^{0}(0), \ldots, z^{N}(0) \in \mathcal{A}$, such that

$$
\operatorname{Rot}\left(z_{i}^{k}(t) ;[0, T]\right)=v_{i} \text { for every } k=0, \ldots, N \text { and } i=1, \ldots, N .
$$

Remark 4.2 It is noted that there is no requirement of uniqueness of Cauchy problems associated to system (4.1) in this higher dimensional Poincaré-Birkhoff theorem for Hamiltonian flows. In $[17,18]$, Theorem 4.1 is applied to prove the multiplicity of periodic solutions of weakly coupled Hamiltonian systems. Theorem 1.1 in the present paper can also be extended to a weakly coupled system of the type

$$
\left\{\begin{array}{l}
x_{i}^{\prime}=f_{i}\left(y_{i}\right)+p_{1 i}(t, x, y) \\
y_{i}^{\prime}=-g_{i}\left(x_{i}\right)+p_{2 i}(t, x, y)
\end{array} \quad(i=1, \ldots, N)\right.
$$

where $x=\left(x_{1}, \ldots, x_{N}\right), y=\left(y_{1}, \ldots, y_{N}\right), f_{i}, g_{i}: \mathbf{R} \rightarrow \mathbf{R}(i=1, \ldots, N)$ are continuous, $p_{j i}$ : $\mathbf{R}^{2 N+1} \rightarrow \mathbf{R}(j=1,2, i=1, \ldots, N)$ are continuous and $2 \pi$-periodic with the variable $t$. Assume that there is a function $U: \mathbf{R}^{2 N+1} \rightarrow \mathbf{R}$ such that

$$
\frac{\partial U}{\partial y_{i}}(t, x, y)=p_{1 i}(t, x, y), \quad \frac{\partial U}{\partial x_{i}}(t, x, y)=-p_{2 i}(t, x, y) \quad(i=1, \ldots, N) .
$$

In this case system (4.2) is a Hamiltonian system. Simple examples of such functions can be given. For example,

$$
U(t, x, y)=p(t) \prod_{i=1}^{i=N} \sin x_{i} \sin y_{i} .
$$

Assume that the following conditions hold: 
$\left(h_{1}^{\prime}\right) g_{i}$ satisfies $\lim _{|u| \rightarrow+\infty} \operatorname{sgn}(u) g_{i}(u)=+\infty, i=1, \ldots, N$;

$\left(h_{2}^{\prime}\right)$ there exist constants $L_{i}>0$ such that, for all $u, v \in \mathbf{R},\left|g_{i}(u)-g_{i}(v)\right| \leq L_{i}|u-v|$, $i=1, \ldots, N$;

$\left(h_{3}^{\prime}\right)$ there are constants $M_{1}>0, M_{2}>0$ and $0<\gamma_{i}<1$ such that $\left|p_{j i}(t, x, y)\right| \leq$ $M_{1}\left(\left|x_{i}\right|+\left|y_{i}\right|\right)^{\gamma_{i}}+M_{2}$ for all $t \in[0,2 \pi],(x, y) \in \mathbf{R}^{2 N}, j=1,2, i=1, \ldots, N ;$

$\left(h_{4}^{\prime}\right)$ there are positive constants $a_{i}$ and $b_{i}$ such that

$$
\lim _{v \rightarrow+\infty} \frac{f_{i}(v)}{v}=a_{i}, \quad \lim _{v \rightarrow-\infty} \frac{f_{i}(v)}{v}=b_{i}
$$

Set $G_{i}(u)=\int_{0}^{u} g_{i}(s) d s, i=1, \ldots, N$. Let us define the time maps

$$
\tau_{i}(c)=\int_{w_{i}(c)}^{d_{i}(c)} \frac{d s}{\sqrt{2\left(c-G_{i}(s)\right)}}
$$

for $c>0$ large enough, where $w_{i}(c)$ and $d_{i}(c)$ satisfy $w_{i}(c)<0<d_{i}(c)$ and $G_{i}\left(w_{i}(c)\right)=$ $G_{i}\left(d_{i}(c)\right)=c$. Assume that the time map $\tau_{i}(c)$ satisfies the condition:

$\left(\tau^{\prime}\right)$ There exist constants $\sigma_{i}>0$, integers $n_{i}>0$, and sequences $\left\{a_{k}^{i}\right\}$ and $\left\{b_{k}^{i}\right\}$ such that $\lim _{k \rightarrow \infty} a_{k}^{i}=+\infty, \lim _{k \rightarrow \infty} b_{k}^{i}=+\infty$; and moreover

$$
\tau_{i}\left(a_{k}^{i}\right)<\frac{2 \pi}{m_{i} n_{i}}-\sigma_{i}, \quad \tau_{i}\left(b_{k}^{i}\right)>\frac{2 \pi}{m_{i} n_{i}}+\sigma_{i}, \quad i=1, \ldots, N,
$$

where $m_{i}=\frac{1}{\sqrt{a_{i}}}+\frac{1}{\sqrt{b_{i}}}$ and $a_{i}, b_{i}$ are given in condition $\left(h_{4}^{\prime}\right)$.

With a slight modification of the proof of Theorem 1.1 and using the higher dimensional Poincaré-Birkhoff Theorem 4.1, we can prove the result.

Theorem 4.3 Assume that conditions $\left(h_{i}^{\prime}\right)(i=1, \ldots, 4)$ and $\left(\tau^{\prime}\right)$ hold. Then Hamiltonian system (4.2) has infinitely many $2 \pi$-periodic solutions $\left\{\left(x^{k}(t), y^{k}(t)\right)\right\}_{k=1}^{\infty}$ which satisfy

$$
\lim _{k \rightarrow \infty}\left(\min _{t \in \mathbf{R}}\left(\left|x^{k}(t)\right|+\left|y^{k}(t)\right|\right)\right)=+\infty
$$

where $\left|x^{k}(t)\right|=\sum_{i=1}^{i=N}\left|x_{i}^{k}(t)\right|,\left|y^{k}(t)\right|=\sum_{i=1}^{i=N}\left|y_{i}^{k}(t)\right|$. Moreover, for each index $i$, both $x_{i}^{k}(t)$ and $y_{i}^{k}(t)$ have exactly $2 n_{i}$ simple zeros in the interval $[0,2 \pi)$.

Competing interests

The authors declare that they have no competing interests.

Authors' contributions

ZW proved Lemma 2.5 and gave the concluding remarks. TM proved the other conclusions and helped to draft the manuscript. All authors read and approved the final manuscript.

\section{Author details}

${ }^{1}$ School of Mathematical Sciences, Capital Normal University, Beijing, 100048, People's Republic of China. ${ }^{2}$ Editorial Department of Journal, Capital Normal University, Beijing, 100048, People's Republic of China.

\section{Acknowledgements}

Research supported by the National Nature Science Foundation of China, No. 11501381 and the Grant of Beijing Education Committee Key Project, No. KZ201310028031.

The authors are grateful to the referees for many valuable suggestions to make the paper more readable. 


\section{Publisher's Note}

Springer Nature remains neutral with regard to jurisdictional claims in published maps and institutional affiliations.

Received: 19 November 2016 Accepted: 23 March 2017 Published online: 04 April 2017

\section{References}

1. Ding, $T$, lannacci, R, Zanolin, F: Existence and multiplicity results for periodic solutions of semilinear Duffing equations. J. Differ. Equ. 105, 364-409 (1993)

2. Ding, T: An infinite class of periodic solutions of periodically perturbed Duffing equations at resonance. Proc. Am. Math. Soc. 86, 47-54 (1982)

3. Hao, D, Ma, S: Semilinear Duffing equations crossing resonance points. J. Differ. Equ. 133, 98-116 (1997)

4. Qian, D: Time maps and Duffing equations across resonant points. Sci. China Ser. A 23, 471-479 (1993)

5. Xia, J, Wang, Z: Existence and multiplicity of periodic solutions for the Duffing equation with singularity. Proc. R. Soc. Edinb., Sect. A 137, 625-645 (2007)

6. Boscaggin, A: Subharmonic solutions of planar Hamiltonian systems: a rotation number approach. Adv. Nonlinear Stud. 11, 77-103 (2011)

7. Boscaggin, $A$, Garrione, M: Resonance and rotation numbers for planar Hamiltonian systems: multiplicity results via the Poincaré-Birkhoff theorem. Nonlinear Anal. 74, 4166-4185 (2011)

8. Fabry, C, Fonda, A: Periodic solutions of perturbed isochronous Hamiltonian systems at resonance. J. Differ. Equ. 214, 299-325 (2005)

9. Fonda, A, Ghirardelli, L: Multiple periodic solutions of Hamiltonian systems in the plane. Topol. Methods Nonlinear Anal. 36, 27-38 (2010)

10. Fonda, A, Sabatini, M, Zanolin, F: Periodic solutions of perturbed Hamiltonian systems in the plane by the use of the Poincaré-Birkhoff theorem. Topol. Methods Nonlinear Anal. 40, 29-52 (2012)

11. Fonda, A, Sfecci, A: A general method for the existence of periodic solutions of differential systems in the plane. J. Differ. Equ. 252, 1369-1391 (2012)

12. Rebelo, C: A note on the Poincaré-Birkhoff fixed point theorem and periodic solutions of planar systems. Nonlinear Anal. 29, 291-311 (1997)

13. Martins, R, Ureña, AJ: The star-shaped condition on Ding's version of the Poincaré-Birkhoff theorem. Bull. Lond. Math. Soc. $39,803-810(2007)$

14. Le Calvez, P, Wang, J: Some remarks on the Poincaré-Birkhoff theorem. Proc. Am. Math. Soc. 138, 703-715 (2010)

15. Ding, W: A generalization of the Poincaré-Birkhoff theorem. Proc. Am. Math. Soc. 88, 341-346 (1983)

16. Fonda, A, Ureña, AJ: A higher dimensional Poincaré-Birkhoff theorem for Hamiltonian flows. Ann. Inst. Henri Poincaré, Anal. Non Linéaire (2016). doi:10.1016/j.anihpc.2016.04.002

17. Calamai, A, Sfecci, A: Multiplicity of periodic solutions for systems of weakly coupled parametrized second order differential equations. Nonlinear Differ. Equ. Appl. (2017). doi:10.1007/s00030-016-0427-5

18. Fonda, A, Sfecci, A: Periodic solutions of weakly coupled super-linear systems. J. Differ. Equ. 260, 2150-2162 (2016)

\section{Submit your manuscript to a SpringerOpen ${ }^{\ominus}$ journal and benefit from:}

- Convenient online submission

- Rigorous peer review

- Immediate publication on acceptance

- Open access: articles freely available online

- High visibility within the field

Retaining the copyright to your article 